Original article

\title{
Joint use of cardio-embolic and bleeding risk scores in elderly patients with atrial fibrillation
}

\author{
Maura Marcucci a,b,*, Alessandro Nobili ${ }^{c}$, Mauro Tettamanti ${ }^{c}$, Alfonso Iorio $^{\mathrm{a}, \mathrm{b}}$, Luca Pasina ${ }^{\mathrm{c}}$, Codjo D. Djade ${ }^{\mathrm{c}}$, \\ Carlotta Franchi ${ }^{\mathrm{c}}$, Alessandra Marengoni ${ }^{\mathrm{d}}$, Francesco Salerno ${ }^{\mathrm{e}}$, Salvatore Corrao ${ }^{\mathrm{f}}$, \\ Francesco Violi $^{\mathrm{g}}$, Pier Mannuccio Mannucci ${ }^{\mathrm{h}}$ on behalf of REPOSI Investigators ${ }^{1}$
}

a Department of Clinical Epidemiology and Biostatistics, McMaster University, Hamilton, ON, Canada

${ }^{\mathrm{b}}$ Department of Medicine, McMaster University, Hamilton, ON, Canada

c IRCCS-Istituto di Ricerche Farmacologiche "Mario Negri”, Milan, Italy

d Geriatric Unit, Ospedali Civili, Department of Medical and Surgery Sciences, University of Brescia, Italy

e Internal Medicine, IRCCS Policlinico San Donato, Department of Medical and Surgery, University of Milan, Italy

${ }^{\mathrm{f}}$ Biomedical Department of Internal Medicine, University of Palermo, Italy

g Divisione I Clinica Medica, Department of Internal Medicine and Medical Specialities, Sapienza University of Rome, Rome, Italy

h Scientific Direction, IRCCS Ca' Granda Maggiore Hospital Foundation, Milan, Italy

\section{A R T I C L E I N F O}

\section{Article history:}

Received 17 May 2013

Received in revised form 16 July 2013

Accepted 9 August 2013

Available online 12 September 2013

\section{Keywords:}

Elderly

Atrial fibrillation

Prediction guides

Bleeding risk

Cardioembolic risk

Thromboprophylaxis

\begin{abstract}
A B S T R A C T
Background: Scores for cardio-embolic and bleeding risk in patients with atrial fibrillation are described in the literature. However, it is not clear how they co-classify elderly patients with multimorbidity, nor whether and how they affect the physician's decision on thromboprophylaxis.

Methods: Four scores for cardio-embolic and bleeding risks were retrospectively calculated for $\geq 65$ year old patients with atrial fibrillation enrolled in the REPOSI registry. The co-classification of patients according to risk categories based on different score combinations was described and the relationship between risk categories tested. The association between the antithrombotic therapy received and the scores was investigated by logistic regressions and CART analyses.

Results: At admission, among 543 patients the median scores (range) were: $\mathrm{CHADS}_{2} 2$ (0-6), $\mathrm{CHA}_{2} \mathrm{DS}_{2}-\mathrm{VASc}_{4}$ (1-9), HEMORR 2 HAGES 3 (0-7), HAS-BLED 2 (1-6). Most of the patients were at high cardio-embolic/high-intermediate bleeding risk (70.5\% combining $\mathrm{CHADS}_{2}$ and HEMORR $2 \mathrm{HAGES,} \mathrm{98.3 \%} \mathrm{combining} \mathrm{CHA}_{2} \mathrm{DS}_{2}-\mathrm{VASc}$ and HAS-BLED). $50-60 \%$ of patients were classified in a cardio-embolic risk category higher than the bleeding risk category. In univariate and multivariable analyses, a higher bleeding score was negatively associated with warfarin prescription, and positively associated with aspirin prescription. The cardio-embolic scores were associated with the therapeutic choice only after adjusting for bleeding score or age.

Conclusion: REPOSI patients represented a population at high cardio-embolic and bleeding risks, but most of them were classified by the scores as having a higher cardio-embolic than bleeding risk. Yet, prescription and type of antithrombotic therapy appeared to be primarily dictated by the bleeding risk.
\end{abstract}

(c) 2013 European Federation of Internal Medicine. Published by Elsevier B.V. All rights reserved.

\section{Introduction}

The underuse of vitamin $\mathrm{K}$ antagonists (VKAs) among elderly patients with atrial fibrillation (AF) has been confirmed in different settings [1-6]. Indeed, the $\mathrm{CHA}_{2} \mathrm{DS}_{2}$-VASc [6,7], that assigns 2 points (and not 1 as $\mathrm{CHADS}_{2}$ [8]) to age $\geq 75$ years, and 1 point to age $\geq 65$ years would qualify all patients older than 75 years as candidates for long term anticoagulation, and all patients older than

\footnotetext{
* Corresponding author at: McMaster University, 1280 Main Street West, Hamilton, ON L8S 4K1, Canada. Tel.: +1905 525 9140x20065; fax: +1905 5268447. E-mail address: Marcucci.maura@gmail.com (M. Marcucci).

1 REPOSI denotes REgistro POliterapie Società Italiana di Medicina Interna.
}

65 years for aspirin treatment, even in the absence of other risk factors [9-11].

Since the fear of treatment-related bleeding is the most likely reason for the under-prescription of anticoagulants, tools for the prediction of the risk of bleeding in patients with AF on VKAs have been proposed [12-15]. All the available scores for bleeding risk include older age among risk factors. The different therapeutic guidelines frame their recommendations on the degree of cardio-embolic risk based upon $\mathrm{CHADS}_{2}$ or $\mathrm{CHA}_{2} \mathrm{DS}_{2}$-VASc, but fail to express uniform agreement on the use and usefulness of bleeding scores, although suggesting of considering the patient bleeding risk to decide on the long-term antithrombotic therapy [9-11]. Moreover, it is still controversial whether and to which extent the decisions on cardio-embolic prophylaxis in the most common population of patients with $\mathrm{AF}$ (the oldest old with 
multimorbidity) currently rely upon a joint assessment of both cardioembolic and bleeding risks.

With this background, we analyze retrospectively patients older than 65 years with atrial fibrillation or flutter (AFF) enrolled in REPOSI [16] during the first (2008) and the second (2010) collection waves of this registry, with the aims to describe: i) the stratification of patients according to the different scores for cardio-embolic and bleeding risks; ii) the within-patient relationship between cardio-embolic and bleeding risks as defined by these scores; iii) whether or not the prescription of antithrombotic therapy was related to the score-based assessment of cardio-embolic and bleeding risks.

\section{Materials and methods}

\subsection{Study population}

Patients analyzed in this study were recruited in the frame of the 'REgistro POliterapie SIMI' (REPOSI) [16]. The REPOSI is a collaborative and independent Registry organized by the Italian Society of Internal Medicine (SIMI) and the Mario Negri Institute of Pharmacological Research in Milan with the purpose to create a network of internal medicine and geriatric wards in order to evaluate hospitalized patients older than 65 years affected by multiple diseases and prescribed with polypharmacy. Patients recruited for REPOSI in 2008 and 2010, and admitted to the participating Italian wards with a known diagnosis of AFF (International Classification of Diseases - Ninth Revision [ICD-9] codes 427.31 or 427.32 ) were analyzed in this study. Patients newly diagnosed with AFF during the index hospitalization were not included.

\subsection{Cardio-embolic and bleeding risk stratification}

The patient population was retrospectively classified according to the cardio-embolic risk as predicted by $\mathrm{CHADS}_{2}$ and $\mathrm{CHA}_{2} \mathrm{DS}_{2}-\mathrm{VASc}$ scores [6,7], and according to the bleeding risk as predicted by HEMORR ${ }_{2}$ HAGES and HAS-BLED scores [12,14]. The components of each score, the annual event rates associated with the risk categories as reported in the literature, as well as the corresponding absolute risk reduction or increase with VKAs are summarized in online Appendix A. The scores were retrospectively calculated for each patient using the data collected at admission on socio-demographic characteristics, clinical history and drug use before the hospitalization and the reason for hospitalization. A modified HEMORR ${ }_{2}$ HAGES score not including genetic risk factors, and a modified HAS-BLED score, not including the labile INR factor were used, because the corresponding data were not available in REPOSI; both these modified versions of the scores have already been used and validated [12,14,17]. The resulting risks were reported both as continuous scores and as categories (low, intermediate, high), using for the latter the originally proposed score-based stratifications $[6,12,14,17,18]$ (online Appendix A). Classification of patients' cardio-embolic risk was compared using both scores, and the classification of patients' bleeding risk using both scores. We then described the co-stratification of the study population using both a scheme for the cardio-embolic risk and one for the bleeding risk, testing different combinations. Correlation between scores, as a measure for trend, was tested by the Spearman test. Concordance/discordance between risk categories was expressed as percentage of patients classified into the same/different risk category. Although risk categories are categorical ordinal variables, linear regression analyses were used to show the average association between the risk categories as defined using the different scores. For this purpose the low, intermediate and high risk categories were coded as 0,1 and 2 , respectively.

\subsection{Antithrombotic therapy and risk scores}

The study population was characterized according to the antithrombotic therapy recorded at hospital admission, considering as long-term therapy VKAs and antiplatelet drugs (aspirin, clopidogrel, ticlopidine and aspirin plus dypyridamole). To evaluate retrospectively the association between the cardio-embolic/bleeding risk scores and the prescribed antithrombotic therapy, two sets of analyses were performed.

a. Risk scores as predictors of VKA prescription. A classic logistic regression was used to evaluate this relationship, in simple and multivariable analyses (including both cardio-embolic and bleeding score as predictors). CART (Classification and Regression Trees analysis) [19] was also used as a multivariable approach to further explore how the scores were hierarchically associated with VKA prescription. The program automatically selected for each score the bestsplitting value for the therapeutic choice, i.e. that value above or below which VKAs were more likely to be prescribed or not.

b. Risk scores as predictors of antithrombotic therapy type. With the aim of taking into account all the possible antithrombotic options for AFF, a 4-level nominal variable was also used as dependent variable, coded as 0 for no therapy, 1 for antiplatelet therapy, 2 for VKAs, and 3 for VKAs plus antiplatelet agents. The variable levels were chosen in order to simulate an ordinal variable where each further level corresponded to an increasing antithrombotic burden. The association between this variable and the scores was explored using an ordered logistic regression when the proportional odds assumption was met, i.e. when the effect of the score on each therapeutic step was constant (the omodel user's command for STATA was used to verify the assumption). If this assumption was not met, a multinomial logistic regression was used, where the no-therapy choice was taken as reference and the association of the score with any other therapeutic choice was compared to the reference.

Then the analyses exploring the association between the risk scores and antithrombotic therapy were repeated adjusting for patient age, in order to look at the effect of the scores after holding the patient age constant; this is equivalent to remove the effect of age (a component of the scores) from the effect of the scores.

In order to take into account the multi-center origin of the REPOSI data, we adopted robust variance estimates that were obtained in all regression models by means of the Huber/White/sandwich estimator which considers observations as independent across groups (the REPOSI centers in this case).

STATA was used to perform all the analyses (version 12, Statacorp, College Station, Tx, US).

\section{Results}

\subsection{Study population}

The 2008-2010 installments of REPOSI included 2712 patients, 1332 enrolled in 2008 and 1380 in 2010. Five hundred and forty-three patients (20.0\%) were admitted to hospital with a known diagnosis of AFF, 247 in 2008 (18.5\%) and 296 in 2010 (21.4\%). Patients with AFF at admission (Table 1) were significantly older than those without (median age $=81.1$, range $65.4-100.6$ years, versus median age $=$ 78.6 , range $65.0-101.4$ years, $\mathrm{p}<0.001$ ); approximately $80 \%$ of patients were older than 75 years. Two hundred sixty-five were males (48.8\%), with no difference in gender composition compared to patients without AFF. Twenty-eight patients with AFF at admission (5\%) died during the hospitalization. Table 1 shows also the proportion of patients presenting a stroke or a bleeding event as reason for admission or during the hospital stay.

\subsection{Cardio-embolic and bleeding risk stratification}

Table 1 reports the mean and median score values at admission. Table 2 shows how the study population was stratified into cardioembolic and bleeding risk categories based upon the different scores. 
Table 1

Demographic and clinical characteristics of the study population. ${ }^{a}$

\begin{tabular}{|c|c|}
\hline \multicolumn{2}{|l|}{ Characteristic } \\
\hline Male, $\mathrm{n}(\%)$ & $265(48.8)$ \\
\hline Mean age \pm SD (median, range) & $81.0 \pm 7.3(81.1,65.4-100.6)$ \\
\hline Median number of drugs per patient (range) & $6(1-15)$ \\
\hline Mean $\mathrm{CHADS}_{2} \pm \mathrm{SD}$ (median, range) & $2.2 \pm 1.1(2,0-6)$ \\
\hline Mean $\mathrm{CHA}_{2} \mathrm{DS}_{2}$-VASc $\pm \mathrm{SD}$ (median, range) & $3.8 \pm 1.2(4,1-9)$ \\
\hline Mean $\mathrm{HEMORR}_{2} \mathrm{HAGES} \pm \mathrm{SD}$ (median, range) & $2.6 \pm 1.2(3,0-7)$ \\
\hline Mean HAS-BLED \pm SD (median, range) & $2.6 \pm 1.1(2,1-6)$ \\
\hline \multicolumn{2}{|l|}{ Oral antithrombotic therapy at admission, $\mathrm{n}(\%)^{\mathrm{b}}$} \\
\hline Vitamin K antagonist & $210(38.7)$ \\
\hline Antiplatelet agent & $174(32.0)$ \\
\hline VKA + antiplatelet & $16(3.0)$ \\
\hline None & $143(26.3)$ \\
\hline Stroke as reason for admission, $\mathrm{n}(\%)$ & $22(4.0)$ \\
\hline Stroke as adverse event during the hospital stay, n (\%) & $2(0.4)$ \\
\hline Bleeding as reason for admission, $\mathrm{n}(\%)$ & $16(2.9)^{\mathrm{C}}$ \\
\hline $\begin{array}{l}\text { Bleeding as adverse event during the hospital stay, } \mathrm{n} \\
(\%)\end{array}$ & $8(1.5)^{c}$ \\
\hline \multicolumn{2}{|c|}{ a The risk scores were calculated counting the risk factors at admission. } \\
\hline \multicolumn{2}{|c|}{$\begin{array}{l}\text { a The risk scores were calculated counting the risk factors at admission. } \\
\text { b } 15 \% \text { of patients not receiving VKAs at admission were on low molecular weight hepa- }\end{array}$} \\
\hline
\end{tabular}

A high correlation was found between the two cardio-embolic risk scores (Spearman correlation coefficient $0.86, \mathrm{p}$ value $<0.001$ ), but with a discordance of $25 \%$ between the two risk classifications. In detail, all patients classified at intermediate or high risk using $\mathrm{CHADS}_{2}$ were classified at high risk according to $\mathrm{CHA}_{2} \mathrm{DS}_{2}$-VASc; patients with a low cardio-embolic risk according to $\mathrm{CHADS}_{2}$ were reclassified by $\mathrm{CHA}_{2} \mathrm{DS}_{2}$-VASc as having an intermediate (9 of $\left.16,56 \%\right)$ or a high ( 7 of $16,43 \%$ ) risk. There was a high correlation between the two bleeding risk scores (Spearman correlation coefficient 0.82 , p value $<0.001$ ), but with a discordance of $43 \%$ between the two risk classifications. In detail, nearly all (117 of 119, 98\%) patients classified at high risk according to HEMORR 2 HAGES were classified at high risk also according to HASBLED; 57\% (193/340) of patients classified at intermediate risk according to HEMORR ${ }_{2}$ HAGES were also classified at intermediate risk according to HAS-BLED, whereas the remaining 43\% patients (147/340) were classified at high HAS-BLED risk. Patients with a low bleeding risk according to HEMORR ${ }_{2}$ HAGES were classified at intermediate (83 among 84, 99\%) or, in one case only, at high HAS-BLED risk. Fig. 1 (plots $\mathrm{a}$ and $\mathrm{b}$ ) in the online Appendix B exemplifies the average relationship between risk categories defined by each couple of scores.

Table 2

Risk stratification according to cardio-embolic and bleeding scores.

\begin{tabular}{|c|c|c|c|c|c|c|}
\hline \multicolumn{7}{|c|}{ a. Cardio-embolic risk scores } \\
\hline \multirow{2}{*}{$\begin{array}{l}\text { Cardio-embolic risk } \\
\text { category }\end{array}$} & \multicolumn{3}{|c|}{$\mathrm{CHADS}_{2}$} & \multicolumn{3}{|c|}{$\mathrm{CHA}_{2} \mathrm{DS}_{2}-\mathrm{VASc}$} \\
\hline & Score & $\begin{array}{l}\text { Number of } \\
\text { patients }\end{array}$ & $\%$ & Score & $\begin{array}{l}\text { Number of } \\
\text { patients }\end{array}$ & $\%$ \\
\hline Low & 0 & 16 & 3.0 & 0 & 0 & 0 \\
\hline Intermediate & 1 & 118 & 21.7 & 1 & 9 & 1.7 \\
\hline High & $\geq 2$ & 409 & 75.3 & $\geq 2$ & 534 & 98.3 \\
\hline \multicolumn{7}{|l|}{ b. Bleeding risk scores } \\
\hline \multirow[t]{2}{*}{ Bleeding risk category } & \multicolumn{3}{|c|}{$\mathrm{HEMORR}_{2} \mathrm{HAGES}$} & \multicolumn{3}{|c|}{ HAS-BLED } \\
\hline & Score & $\begin{array}{l}\text { Number of } \\
\text { patients }\end{array}$ & $\%$ & Score & $\begin{array}{l}\text { Number of } \\
\text { patients }\end{array}$ & $\%$ \\
\hline Low & $0-1$ & 84 & 15.5 & 0 & 0 & 0 \\
\hline Intermediate & $2-3$ & 340 & 62.6 & $1-2^{\mathrm{a}}$ & 278 & 51.2 \\
\hline High & $\geq 4$ & 119 & 21.9 & $\geq 3$ & 265 & 48.8 \\
\hline
\end{tabular}

a 60 patients (11.0\%) had a HAS-BLED score 1 .
Table 3

Patient distribution according to cardio-embolic and bleeding risk categories: number of patients (\% of the whole population).

\begin{tabular}{|c|c|c|c|c|}
\hline $\mathrm{CHADS}_{2} \mathrm{HEMORR}_{2} \mathrm{HAGES}$ & Low risk & $\begin{array}{l}\text { Intermediate } \\
\text { risk }\end{array}$ & High risk & Total \\
\hline Low risk & $11(2.0)$ & $5(0.9)$ & - & \\
\hline Intermediate risk & $47(8.7)$ & $62(11.4)$ & $9(1.7)$ & \\
\hline High risk & $26(4.8)$ & $273(50.3)$ & $110(20.2)$ & \\
\hline & & & & $543(100)$ \\
\hline
\end{tabular}

\begin{tabular}{|c|c|c|c|c|}
\hline $\mathrm{CHA}_{2} \mathrm{DS}_{2}-\mathrm{VASC}$ & Low risk & $\begin{array}{l}\text { Intermediate } \\
\text { risk }\end{array}$ & High risk & Total \\
\hline Low risk & - & - & - & \\
\hline Intermediate risk & - & $8(1.5)$ & $1(0.2)$ & \\
\hline High risk & - & $270(49.7)$ & $264(48.6)$ & \\
\hline & & & & $543(100)$ \\
\hline
\end{tabular}

According to the predicted risk associated with the scores reported in the original papers (see the online Appendix A for details): White cells: the predicted annualized cardioembolic risk tends to be larger than the predicted annualized bleeding risk (and the predicted absolute risk reduction of cardio-embolic events with warfarin tends to be larger than the predicted absolute risk increase of bleeding events with warfarin). Dark gray cells: the predicted annualized bleeding risk tends to be larger than the predicted annualized cardio-embolic risk (and the predicted absolute risk increase of bleeding events with warfarin tends to be larger than the predicted absolute risk reduction of cardio-embolic events with warfarin). Light gray cells: the predicted annualized bleeding risk tends to be equal or larger than the predicted annualized cardio-embolic risk (and the predicted absolute risk increase of bleeding events with warfarin tends to be equal or larger than the predicted absolute risk reduction of cardio-embolic events with warfarin). Predicted denotes as reported in score validation studies.

Table 3 shows how the study population was co-classified according to both the cardio-embolic and bleeding risks using two different score combinations. The Spearman correlation between $\mathrm{CHADS}_{2}$ and HEMORR ${ }_{2}$ HAGES scores and between $\mathrm{CHA}_{2} \mathrm{DS}_{2}$-VASc and HAS-BLED scores was, respectively, 0.424 and 0.316 . Most of the patients were at high cardio-embolic/high-intermediate bleeding risk (70.5\% when $\mathrm{CHADS}_{2}$ plus HEMORR 2 HAGES were used, $98.3 \%$ when $\mathrm{CHA}_{2} \mathrm{DS}_{2}-\mathrm{VASC}$ plus HAS-BLED were used). Plots $c$, $d$, e and $\mathrm{f}$ in Fig. 1 (online Appendix A) show the average relationship between cardio-embolic and bleeding risk categories using the 4 possible score combinations.

\subsection{Antithrombotic therapy and risk scores}

The antithrombotic therapy that REPOSI patients were receiving at admission is shown in Table 1.

\subsubsection{Risk scores as predictors of VKA prescription}

Table 4 reports the number and percentage of patients on VKAs in each cell co-defined by the cardio-embolic and bleeding risk. The highest rate of VKA prescription was found among patients at intermediate cardio-embolic and low bleeding risk when the $\mathrm{CHADS}_{2}$ / HEMORR $_{2}$ HAGES co-classification was used, and among patients at high cardio-embolic and intermediate bleeding risk when the $\mathrm{CHA}_{2} \mathrm{DS}_{2}$-VASc/HAS-BLED combination was used (ignoring the $100 \%$ cell including only 1 patient). In simple logistic regressions, a higher bleeding score, using either HEMORR ${ }_{2}$ HAGES or HAS-BLED, was associated with a lower probability to receive VKA ( $p<0.001)$. Neither cardio-embolic risk score was significantly associated with VKAs prescription in unadjusted analysis. Only after adjusting for the bleeding risk score (either HEMORR ${ }_{2}$ HAGES or HAS-BLED) was a higher cardioembolic risk score (either $\mathrm{CHADS}_{2}$ or $\mathrm{CHA}_{2} \mathrm{DS}_{2}$-VASc) associated with a higher probability to receive VKAs ( $\mathrm{p}<0.001$ for any combination). When all the 4 scores were included as covariates, the HEMORR ${ }_{2}$ HAGES 
Table 4

Frequency of VKA prescription according to cardio-embolic and bleeding risk categories: number of patients (\% of the total number of patients in each cell).

\begin{tabular}{|c|c|c|c|c|}
\hline HEMORR $_{2}$ HAGES & Low risk & $\begin{array}{c}\text { Intermediate } \\
\text { risk }\end{array}$ & High risk & \\
\hline Low risk & $4(36.4)$ & $1(20.0)$ & - & $5(31.2)$ \\
\hline Intermediate risk & $31(66.0)$ & $19(30.6)$ & $0(0.0)$ & $40(33.9)$ \\
\hline High risk & $16(61.5)$ & $130(47.6)$ & $25(22.7)$ & $171(41.8)$ \\
\hline
\end{tabular}

\begin{tabular}{|c|c|c|c|c|}
\hline HAS-BLED & Low risk & $\begin{array}{c}\text { Intermediate } \\
\text { risk }\end{array}$ & High risk & \\
\hline Low risk & - & - & - & - \\
\hline Intermediate risk & - & $2(25.0)$ & $1(100.0)$ & $3(33.3)$ \\
\hline High risk & - & $150(55.6)$ & $73(27.6)$ & $223(41.8)$ \\
\hline
\end{tabular}

According to the predicted risk associated with the scores reported in the original papers (see the online Appendix A for details): White cells: the predicted annualized cardioembolic risk tends to be larger than the predicted annualized bleeding risk (and the predicted absolute risk reduction of cardio-embolic events with warfarin tends to be larger than the predicted absolute risk increase of bleeding events with warfarin). Dark gray cells: the predicted annualized bleeding risk tends to be larger than the predicted annualized cardio-embolic risk (and the predicted absolute risk increase of bleeding events with warfarin tends to be larger than the predicted absolute risk reduction of cardio-embolic events with warfarin). Light gray cells: the predicted annualized bleeding risk tends to be equal or larger than the predicted annualized cardio-embolic risk (and the predicted absolute risk increase of bleeding events with warfarin tends to be equal or larger than the predicted absolute risk reduction of cardio-embolic events with warfarin). Predicted denotes as reported in score validation studies.

and $\mathrm{CHADS}_{2}$ scores remained significant predictors. The CART analysis confirmed these results, and pointed out that a low bleeding risk score seemed to affect positively the probability of VKA prescription, whereas cardio-embolic risk scores were associated with the probability of VKA prescription only among higher bleeding risk scores (Fig. 2 in the online Appendix A).

\subsubsection{Risk scores as predictors of the type of antithrombotic therapy}

When an ordered 4-level variable was used for antithrombotic therapy, the proportional odds assumption was met for both cardio-embolic risk scores, i.e. higher scores were associated to therapeutic choices with a higher antithrombotic potency, but in a quasi statistically significant way only for $\mathrm{CHADS}_{2}(\mathrm{p}=0.054)$. The proportional odds assumption was not met for the bleeding risk scores. In simple multinomial analysis, and also after adjusting for any cardio-embolic risk score, the HEMORR HAGES score was associated with the therapeutic choice, but in different ways: a higher HEMORR ${ }_{2}$ HAGES score was negatively associated with the prescription of VKA compared to no therapy, but it was positively associated with the prescription of antiplatelet agents compared to no therapy. A direct association between a higher score and antiplatelet prescription was also found for the HAS-BLED score in simple multinomial logistic regression, and after adjusting for any cardio-embolic risk score. Conversely HAS-BLED was not associated with the prescription of VKA. None of the reported findings changed when patients on LMWH or fondaparinux were excluded from the analyses. After adjusting for patient age, both the cardio-embolic risk scores became significantly associated with the antithrombotic therapy in all types of analysis even without adjusting for the bleeding risk scores. All the remaining results did not change.

\section{Discussion}

The REPOSI registry was designed in order to collect data on a representative sample of patients admitted to internal medicine wards, increasingly characterized in Italy and elsewhere in Europe by advanced age and multimorbidity. The first aim of these post-hoc analyses was to describe how the available scores for cardio-embolic and bleeding risks would classify patients with AFF in this complex population. We then evaluated whether or not risk assessment according to the scores was related the choice of antithrombotic therapy.

The main novelty of this study was to look, albeit retrospectively, at the co-classification of this elderly population using a combination of scores for both cardio-embolic and bleeding risk, that might theoretically provide the physician with a higher potential for tailoring each individual treatment than using a strategy based only on the cardioembolic. As expected, the REPOSI population was on average both at high cardio-embolic and bleeding risk (see Table 2a), even though the patients' cardio-embolic risk category tended to be higher than the bleeding risk category. In particular, the percentage of patients belonging to a cardio-embolic risk category higher than the bleeding risk category was more than $60 \%$ when $\mathrm{CHADS}_{2}$ plus HEMORR ${ }_{2} \mathrm{HAGES}$ were jointly used, and approximately $50 \%$ when $\mathrm{CHA}_{2} \mathrm{DS}_{2}$-VASc plus HASBLED were used. This co-classification would apparently lead to recommend anticoagulation for approximately $50 \%$ of REPOSI patients. However, the same definitions for risk category (i.e. low, intermediate or high) for different scores do not correspond to the same annual risk of stroke or bleeding (and so to the same absolute effect of the treatment), as reported in the online Appendix A. In addition, a more appropriate way of using the score-based predictions of risk to individualize treatment recommendations should take into account also the different weight that a patient might assign to such clinical events, as stroke and bleeding [20-22].

Our data confirm the well-known reclassification effect of $\mathrm{CHA}_{2} \mathrm{DS}_{2}-$ VASc $[6,7,19]$, which moved almost all patients at low and intermediate $\mathrm{CHADS}_{2}$ score to the high risk category. As expected by definition for a $\geq 65$ year population, none of the REPOSI patients was classified as having a low $\mathrm{CHA}_{2} \mathrm{DS}_{2}-\mathrm{VASc}$ risk $[7,19]$, with the implication that according to this score all REPOSI patients with AFF would be treated with anticoagulants.

The cardio-embolic risk stratification of REPOSI patients resembled that recently described in an elderly cohort from the UK General Practice Research Database (GPRD) [5]. However, the REPOSI population had a higher representation of patients at intermediate-high $\mathrm{CHADS}_{2}$ score, presumably because of a higher mean age and different selection criteria (patients at the time of hospital admission, with a likely higher rate of morbidity than those referred to general practitioners).

There was also a high representation of the high risk category for bleeding among REPOSI patients, higher than in the UK cohort [5]. As for the cardio-embolic scores, a reclassification effect with HAS-BLED was observed compared to HEMORR ${ }_{2}$ HAGES. Indeed, none of the REPOSI patients was at low HAS-BLED risk (because of age, none had a 0 score), and in $40 \%$ of them HAS-BLED classified patients into a higher risk category than HEMORR ${ }_{2}$ HAGES. This effect was attenuated provided that a HAS-BLED score of 1 was included in the low risk category together with score 0 (as done in other studies $[5,18]$ ), yet only $11 \%$ of patients had a HAS-BLED score of 1. In fact, HAS-BLED was developed in order to provide a therapeutic guideline easier to memorize and includes more practicable risk factors than HEMORR ${ }_{2} \mathrm{HAGES} \mathrm{[14].}$

We observed a low overall rate of prescription of VKAs, confirming a previous analysis based on REPOSI [16]. More interesting, the distribution of the percentages of patients treated with VKAs across the cells defined by the scores (Table 3 ) and the results of the logistic analyses showed that the patient's bleeding risk, but not the cardio-embolic risk alone, predicted the therapeutic choice. These findings on the relationship between the bleeding score and VKA prescription are consistent with those of the UK cohort [5]. In the literature, evidence on the relationship between cardio-embolic scores and VKA prescription in real settings is not uniform $[5,23,24]$. In the present study, the cardioembolic risk was a predictor of VKA prescription, only after adjusting for the bleeding score or patient age. In addition, the association found between a higher bleeding score and antiplatelet therapy clearly 
confirms the tendency to prescribe aspirin in clinical practice when evidence or perception of a higher risk of bleeding prevents VKA prescription. Irrespective of the cardio-embolic risk, this situation materializes especially in the elderly, even though this behavior is not justified by a safer profile of aspirin compared to VKAs [25,26], and either by a clear efficacy of aspirin [27].

This study has several limitations. First, a certain degree of underreporting is expected because of the post-hoc nature of our research question. Thus, it is possible that the actual risk scores were underestimated. Second, this was only an indirect and theoretical investigation of the association between patients' risks and physician's decisions, because it is not known whether or not REPOSI physicians applied these scores to take decisions. Another limitation is the assumption that the risk scores proposed in the literature for patients with AFF have a good predictive ability in a REPOSI-like elderly population. A further fundamental step should be the evaluation of the impact on patient outcomes of a decision strategy based on combined cardio-embolic and bleeding risk assessment compared to a strategy of cardioembolic risk assessment alone.

\section{Learning points}

- Scores for cardio-embolic and bleeding risk in patients with atrial fibrillation are described in the literature to aid at tailoring the long term antithrombotic therapy; all of them include age as risk factor.

- We observed how the available scores $\left(\mathrm{CHADS}_{2}\right.$ and $\mathrm{CHA}_{2} \mathrm{DS}_{2}-\mathrm{VASc}$, for cardio-embolic risk, and HEMORR ${ }_{2}$ HAGES and HAS-BLED, for bleeding risk) co-classified complex elderly patients with multimorbidity admitted to Italian internal medicine and geriatric wards, and we confirmed that they configured a population both at high cardio-embolic and at high bleeding risk.

- $50-60 \%$ of patients (depending on the score couples used) were classified in a cardio-embolic risk category higher than the bleeding risk category.

- In those patients, the prescription and the type of antithrombotic therapy appeared to be primarily influenced by the bleeding risk; both the cardio-embolic scores were associated with the therapeutic choice only after adjusting for the patient bleeding score or age.

\section{Conflict of interest}

The authors declare that they do not have any conflict of interests.

\section{Acknowledgments}

We thank Prof. John C. Sinclair at the Department of Clinical Epidemiology and Biostatistics, McMaster University, for the intellectual stimulus, the support and the help provided during the draft of the manuscript.

Investigators and co-authors of the REPOSI (REgistro POliterapie

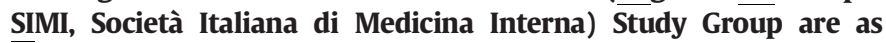
follows

Steering Committee: Pier Mannuccio Mannucci (Chair, Fondazione IRCCS Cà Granda Ospedale Maggiore Policlinico, Milano), Alessandro Nobili (co-chair, Istituto di Ricerche Farmacologiche "Mario Negri", Milano), Mauro Tettamanti, Luca Pasina, Carlotta Franchi (Istituto di Ricerche Farmacologiche "Mario Negri", Milano), Francesco Salerno (IRCCS Policlinico San Donato Milanese, Milano), Salvatore Corrao (ARNAS Civico, Di Cristina, Benfratelli, DiBiMIS, Università di Palermo, Palermo), Alessandra Marengoni (Spedali Civili di Brescia, Brescia), Alfonso Iorio (McMaster University, Hamilton, Canada), Maura Marcucci (McMaster University, Hamilton, Canada).
Clincal data monitoring and revision: Valentina Spirito, Damia Noce, Jacopo Bonazzi, Rossana Lombardo, Eleonora Sparacio, Stefania Alborghetti (Istituto di Ricerche Farmacologiche "Mario Negri", Milano).

Database Management and Statistics: Mauro Tettamanti, Luigi De Vittorio, Codjo Djignefa Djade (Istituto di Ricerche Farmacologiche "Mario Negri", Milano).

Investigators: Domenico Prisco, Elena Silvestri, Caterina Cenci, Tommaso Barnini (Azienda Ospedaliero Universitaria Careggi Firenze, SOD Patologia Medica); Giuseppe Delitala, Stefano Carta, Sebastiana Atzori (Azienda Mista Ospedaliera Universitaria, Sassari, Clinica Medica); Gianfranco Guarnieri, Michela Zanetti, Annalisa Spalluti (Azienda Ospedaliera Universitaria Ospedali Riuniti di Trieste, Trieste, Clinica Medica Generale e Terapia Medica); Maria Grazia Serra, Maria Antonietta Bleve (Azienda Ospedaliera "Cardinale Panico" di Tricase, Lecce, Unità Operativa Complessa Medicina); Massimo Vanoli, Giulia Grignani, Gianluca Casella (Azienda Ospedaliera della Provincia di Lecco, Ospedale di Merate, Lecco, Medicina Interna); Laura Gasbarrone (Azienda Ospedaliera Ospedale San Camillo Forlanini, Roma, Medicina Interna 1); Giorgio Maniscalco, Massimo Gunelli, Daniela Tirotta (Azienda Ospedaliera Ospedale San Salvatore, Pesaro, Soc Medicina Interna); Antonio Brucato, Silvia Ghidoni, Paola Di Corato (Azienda Ospedaliera Papa Giovanni XXIII, Bergamo, Medicina 1); Mauro Bernardi, Silvia Li Bassi, Luca Santi (Azienda Ospedaliera Policlinico Sant'Orsola-Malpighi, Bologna, Semeiotica Medica Bernardi); Giancarlo Agnelli, Alfonso Iorio, Maura Marcucci, Emanuela Marchesini (Azienda Ospedaliera Santa Maria della Misericordia, Perugia, Medicina Interna e Cardiovascolare); Elmo Mannarino, Graziana Lupattelli, Pamela Rondelli, Francesco Paciullo (Azienda Ospedaliera Santa Maria della Misericordia, Perugia, Medicina Interna, Angiologia, Malattie da Arteriosclerosi); Fabrizio Fabris, Michela Carlon, Francesca Turatto (Azienda Ospedaliera Università di Padova, Padova, Clinica Medica I); Maria Cristina Baroni, Marianna Zardo (Azienda Ospedaliera Università di Parma, Parma, Clinica e Terapia Medica); Roberto Manfredini, Christian Molino, Marco Pala, Fabio Fabbian (Azienda Ospedaliera - Universitaria Sant'Anna, Ferrara, Unità Operativa Clinica Medica); Ranuccio Nuti, Roberto Valenti, Martina Ruvio, Silvia Cappelli (Azienda Ospedaliera Università Senese, Siena, Medicina Interna I); Giuseppe Paolisso, Maria Rosaria Rizzo, Maria Teresa Laieta (Azienda Ospedaliera Universitaria della Seconda Università degli Studi di Napoli, Napoli, VI Divisione di Medicina Interna e Malattie Nutrizionali dell'Invecchiamento); Teresa Salvatore, Ferdinando Carlo Sasso (Azienda Ospedaliera Universitaria della Seconda Università degli Studi di Napoli, Napoli, Medicina Interna e Malattie Epato-Bilio Metaboliche Avanzate); Riccardo Utili, Emanuele Durante Mangoni, Daniela Pinto (Azienda Ospedaliera Universitaria della Seconda Università degli Studi di Napoli, Napoli, Medicina Infettivologica e dei trapianti); Oliviero Olivieri, Anna Maria Stanzial (Azienda Ospedaliera Universitaria Integrata di Verona, Verona, Unità Operativa di Medicina Interna B); Renato Fellin, Stefano Volpato, Sioulis Fotini (Azienda Ospedaliera Universitaria Ospedale Sant'Anna, Ferrara, Unità Operativa di Medicina Interna Gerontologia e Geriatria); Mario Barbagallo, Ligia Dominguez, Lidia Plances, Daniela D'Angelo (Azienda Ospedaliera Universitaria Policlinico Giaccone Policlinico di Palermo, Palermo, Unità Operativa di Geriatria e Lungodegenza); Giovanbattista Rini, Pasquale Mansueto, Ilenia Pepe (Azienda Ospedaliera Universitaria Policlinico P. Giaccone di Palermo, Palermo, Medicina Interna e Malattie Metaboliche); Giuseppe Licata, Luigi Calvo, Maria Valenti (Azienda Ospedaliera Universitaria Policlinico P. Giaccone di Palermo, Palermo, Medicina Interna e Cardioangiologia); Claudio Borghi, Enrico Strocchi, Elisa Rebecca Rinaldi (Azienda Ospedaliera Universitaria Policlinico S. Orsola-Malpighi, Bologna, Unità Operativa di Medicina Interna Borghi); Marco Zoli, Elisa Fabbri, Donatella Magalotti (Azienda Ospedaliera Universitaria Policlinico S. OrsolaMalpighi, Bologna, Unità Operativa di Medicina Interna Zoli); Alberto Auteri, Anna Laura Pasqui, Luca Puccetti (Azienda Ospedaliera Universitaria Senese, Siena, Medicina 3); Franco Laghi Pasini, Pier Leopoldo Capecchi, Maurizio Bicchi (Azienda Ospedaliera Universitaria Senese, Siena, Unità Operativa Complessa Medicina 2); Carlo Sabbà, 
Francesco Saverio Vella, Alessandro Marseglia, Chiara Valentina Luglio (Azienda Ospedaliero-Universitaria Consorziale Policlinico di Bari, Bari, Medicina Interna Universitaria C. Frugoni); Giuseppe Palasciano, Maria Ester Modeo, Annamaria Aquilino, Pallante Raffaele (Azienda Ospedaliero-Universitaria Consorziale Policlinico di Bari, Bari, Medicina Interna Ospedale "Pende-Ferrannini"); Stefania Pugliese, Caterina Capobianco (Azienda Ospedaliero-Universitaria Consorziale Policlinico di Bari, Bari, Clinica Medica I Augusto Murri); Alfredo Postiglione, Maria Rosaria Barbella, Francesco De Stefano (Azienda Ospedaliera Universitaria Policlinico Federico II di Napoli, Medicina Geriatrica Dipartimento di Clinica Medica); Luigi Fenoglio, Chiara Brignone, Christian Bracco, Alessia Giraudo (Azienda Sanitaria Ospedaliera Santa Croce e Carle di Cuneo, Cuneo, S. C. Medicina Interna); Giuseppe Musca, Olga Cuccurullo (Azienda Sanitaria Provinciale di Cosenza Presidio Ospedaliero di Cetraro, Cosenza, Unità Operativa Complessa Medicina Interna); Luigi Cricco, Alessandra Fiorentini ( $С O B$ Stabilimento Montefiascone, Viterbo, Unità Operativa Complessa di Geriatria e Medicina); Maria Domenica Cappellini, Giovanna Fabio, Sonia Seghezzi, Margherita Migone De Amicis (Fondazione IRCCS Cà Granda Ospedale Maggiore Policlinico, Milano, Unità Operativa Medicina Interna IA); Silvia Fargion, Paola Bonara, Mara Bulgheroni, Rosa Lombardi (Fondazione IRCCS Cà Granda Ospedale Maggiore Policlinico, Milano, Medicina Interna 1B); Fabio Magrini, Ferdinando Massari, Tatiana Tonella (Fondazione IRCCS Cà Granda Ospedale Maggiore Policlinico, Milano, Unità Operativa Medicina Cardiovascolare); Flora Peyvandi, Alberto Tedeschi, Raffaella Rossio (Fondazione IRCCS Cà Granda Ospedale Maggiore Policlinico, Milano, Medicina Interna 2); Guido Moreo, Barbara Ferrari, Luisa Roncari (Fondazione IRCCS Cà Granda Ospedale Maggiore Policlinico, Milano, Medicina Interna 3); Valter Monzani, Valeria Savojardo, Christian Folli, Maria Magnini (Fondazione IRCCS Cà Granda Ospedale Maggiore Policlinico, Milano, Medicina d'Urgenza); Daniela Mari, Paolo Dionigi Rossi, Sarah Damanti, Silvia Prolo (Fondazione IRCCS Cà Granda Ospedale Maggiore Policlinico, Milano, Geriatria); Maria Sole Lilleri (Fondazione IRCCS Cà Granda Ospedale Maggiore Policlinico, Milano, Medicina Generale ad Indirizzo Geriatrico); Luigi Cricco, Alessandra Fiorentini (COB Viterbo, Stabilimento Montefiascone, Viterbo, UOC Geriatria e Medicina); Giuliana Micale (IRCCS Istituto Auxologico Italiano, Milano, Medicina Generale ad indirizzo Geriatrico); Mauro Podda, Carlo Selmi, Francesca Meda (IRCCS Istituto Clinico Humanitas, Milano, Clinica Medica); Francesco Salerno, Silvia Accordino, Alessio Conca, Valentina Monti (IRCCS Policlinico San Donato e Università di Milano, San Donato Milanese, Medicina Interna); Gino Roberto Corazza, Emanuela Miceli, Marco Vincenzo Lenti, Donatella Padula (IRCCS Policlinico San Matteo di Pavia, Pavia, Clinica Medica I, Reparto 11); Carlo L. Balduini, Giampiera Bertolino, Stella Provini, Federica Quaglia (IRCCS Policlinico San Matteo di Pavia, Pavia, Clinica Medica III); Giovanni Murialdo, Marta Bovio (IRCS Azienda Ospedaliera Universitaria San Martino-IST di Genova, Genova, Clinica di Medicina Interna 2); Franco Dallegri, Luciano Ottonello, Alessandra Quercioli, Alessandra Barreca (Università di Genova, Genova, Medicina Interna 1); Maria Beatrice Secchi, Davide Ghelfi (Ospedale Bassini di Cinisello Balsamo, Milano, Divisione Medicina); Wu Sheng Chin, Laura Carassale, Silvia Caporotundo (Ospedale Bassini, Cinisello Balsamo, Milano, Unità Operativa di Geriatria); Luigi Anastasio, Lucia Sofia, Maria Carbone (Ospedale Civile Jazzolino di Vibo Valentia, Vibo Valentia, Medicina interna); Giancarlo Traisci, Lucrezia De Feudis, Silvia Di Carlo (Ospedale Civile Santo Spirito di Pescara, Pescara, Medicina Interna 2); Giovanni Davì, Maria Teresa Guagnano, Simona Sestili (Ospedale Clinicizzato SS. Annunziata, Chieti, Clinica Medica); Elisabetta Bergami, Emanuela Rizzioli (Ospedale del Delta, Lagosanto, Ferrara, Medicina Interna); Carlo Cagnoni, Luca Bertone, Antonio Manucra (Ospedale di Bobbio, Piacenza, Unità Operativa Medicina e Primo Soccorso); Alberto Buratti, Tiziana Tognin, Nicola Lucio Liberato (Azienda Ospedaliera della Provincia di Pavia, Ospedale di Casorate Primo, Pavia, Medicina Interna); Giordano Bernasconi, Barbara Nardo (Ospedale di Circolo di Busto Arsizio, Varese, Medicina I); Giovanni Battista Bianchi, Sabrina Giaquinto Ospedale "SS Gerosa e Capitanio" di Lovere, Bergamo, Unità Operativa
Complessa di Medicina Generale, Azienda Ospedaliera "Bolognini" di Seriate, Bergamo; Giampiero Benetti, Michela Quagliolo, Giuseppe Riccardo Centenaro (Ospedale di Melegnano, Vizzolo Predabissi, Melegnano, Medicina 1); Francesco Purrello, Antonino Di Pino, Salvatore Piro (Ospedale Garibaldi Nesima, Catania, Unità Operativa Complessa di Medicina Interna); Gerardo Mancuso, Daniela Calipari, Mosè Bartone, Francesco Gullo (Ospedale Giovanni Paolo II Lamezia Terme, Catanzaro, Unità Operativa Complessa Medicina Interna); Michele Cortellaro, Marina Magenta, Francesca Perego; Maria Rachele Meroni (Ospedale Luigi Sacco, Milano, Medicina $3^{\circ}$ ); Marco Cicardi, Antonio Gidaro Marina Magenta (Ospedale Luigi Sacco, Milano, Medicina II); Andrea Sacco, Antonio Bonelli, Gaetano Dentamaro (Ospedale Madonna delle Grazie, Matera, Medicina); Renzo Rozzini, Lina Falanga, Alessandro Giordano (Ospedale Poliambulanza, Brescia, Medicina Interna e Geriatria); Paolo Cavallo Perin, Bartolomeo Lorenzati, Gabriella Gruden, Graziella Bruno (Dipartimento di Scienze Mediche, Università di Torino, Città della Scienza e della Salute, Torino, Medicina 3); Giuseppe Montrucchio, Elisabetta Greco, Pietro Tizzani (Dipartimento di Scienze Mediche, Università di Torino, Città della Scienza e della Salute, Torino, Medicina Interna 5); Giacomo Fera, Maria Loreta Di Luca, Donatella Renna (Ospedale San Giacomo di Monopoli, Bari, Unità Operativa Medicina Interna); Antonio Perciccante, Alessia Coralli (Ospedale San Giovanni-Decollato-Andisilla, Civita Castellana Medicina); Rodolfo Tassara, Deborah Melis, Lara Rebella (Ospedale San Paolo, Savona, Medicina I); Giorgio Menardo, Stefania Bottone, Elsa Sferrazzo (Ospedale San Paolo, Savona, Medicina Interna e Gastroenterologia); Claudio Ferri, Rinaldo Striuli, Rosa Scipioni (Ospedale San Salvatore, L'Aquila, Medicina Interna Universitaria); Raffaella Salmi, Piergiorgio Gaudenzi, Susanna Gamberini, Franco Ricci (Azienda Ospedaliera-Universitaria S. Anna, Ferrara, Unità Operativa di Medicina Ospedaliera II); Cosimo Morabito, Roberto Fava (Ospedale Scillesi d'America, Scilla Medicina); Andrea Semplicini, Lucia Gottardo (Ospedale SS. Giovanni e Paolo, Venezia, Medicina Interna 1); Giuseppe Delitala, Stefano Carta, Sebastiana Atzori (Ospedale Universitario Policlinico di Sassari, Sassari, Clinica Medica); Gianluigi Vendemiale, Gaetano Serviddio, Roberta Forlano (Ospedali Riuniti di Foggia, Foggia, Medicina Interna Universitaria); Luigi Bolondi, Leonardo Rasciti, Ilaria Serio (Policlinico Sant'Orsola-Malpighi, Bologna, Unità Operativa Complessa Medicina Interna); Cesare Masala, Antonio Mammarella, Valeria Raparelli (Policlinico Umberto I, Roma, Medicina Interna D); Filippo Rossi Fanelli, Massimo Delfino, Antonio Amoroso (Policlinico Umberto I, Roma, Medicina Interna $\mathrm{H}$ ); Francesco Violi, Stefania Basili, Ludovica Perri (Policlinico Umberto I, Roma, Prima Clinica Medica); Pietro Serra, Vincenzo Fontana, Marco Falcone (Policlinico Umberto I, Roma, Terza Clinica Medica); Raffaele Landolfi, Antonio Grieco, Antonella Gallo (Policlinico Universitario A. Gemelli, Roma, Clinica Medica); Giuseppe Zuccalà, Francesco Franceschi, Guido De Marco, Cordischi Chiara, Sabbatini Marta (Policlinico Universitario A. Gemelli, Roma, Roma, Unità Operativa Complessa Medicina d'Urgenza e Pronto Soccorso); Martino Bellusci, Donatella Setti, Filippo Pedrazzoli (Presidio Ospedaliero Alto Garda e Ledro, Ospedale di Arco, Trento, Unità Operativa di Medicina Interna Urgenza/Emergenza); Giuseppe Romanelli, Caterina Pirali, Claudia Amolini (Spedali Civili di Brescia, Brescia, Geriatria); Enrico Agabiti Rosei, Damiano Rizzoni, Luana Castoldi (Spedali Civili di Brescia, Brescia, Seconda Medicina); Antonio Picardi, Umberto Vespasiani Gentilucci, Chiara Mazzarelli, Paolo Gallo (Università Campus BioMedico, Roma, Medicina Clinica-Epatologia); Luigina Guasti, Luana Castiglioni, Andrea Maresca, Alessandro Squizzato, Sara Contini, Marta Molaro (Università degli Studi dell'Insubria, Ospedale di Circolo e Fondazione Macchi, Varese, Medicina Interna I); Giorgio Annoni, Maurizio Corsi Sara Zazzetta (Università degli studi di Milano-Bicocca Ospedale S. Gerardo, Monza, Unità Operativa di Geriatria; Marco Bertolotti, Chiara Mussi Roberto Scotto, Maria Alice Ferri, Francesca Veltri (Università di Modena e Reggio Emilia, AUSL di Modena, Modena, Nuovo Ospedale Civile, Unità Operativa di Geriatria); Franco Arturi, Elena Succurro, Giorgio Sesti, Umberto Gualtieri (Università degli Studi Magna Grecia, Policlinico Mater Domini, Catanzaro, Unità Operativa Complessa di Medicina Interna); 
Francesco Perticone, Angela Sciacqua, Michele Quero, Chiara Bagnato (Università Magna Grecia Policlinico Mater Domini, Catanzaro, Unità Operativa Malattie Cardiovascolari Geriatriche); Paola Loria, Maria Angela Becchi, Gianfranco Martucci, Alessandra Fantuzzi, Mauro Maurantonio (Università di Modena e Reggio Emilia, Medicina Metabolica-NOCSAE, Baggiovara, Modena); Roberto Corinaldesi, Roberto De Giorgio, Mauro Serra, Valentina Grasso, Eugenio Ruggeri, Lorenzo Mauro Carozza, Fabio Pignatti (Dipartimento di Scienze Mediche e Chirurgiche, Unità Operativa di Medicina Interna, Università degli Studi di Bologna/Azienda Ospedaliero-Universitaria S. Orsola-Malpighi, Bologna).

\section{Appendix A. Supplementary data}

Supplementary data to this article can be found online at http://dx. doi.org/10.1016/j.ejim.2013.08.697.

\section{References}

[1] McCormick D, Gurwitz JH, Goldberg RJ, Becker R, Tate JP, Elwell A, et al. Prevalence and quality of warfarin use for patients with atrial fibrillation in the long-term care setting. Arch Intern Med 2001;161:2458-63.

[2] Waldo AL, Becker RC, Tapson VF, Colgan KJ. NABOR Steering Committee. Hospitalized patients with atrial fibrillation and a high risk of stroke are not being provided with adequate anticoagulation. J Am Coll Cardiol 2005;46:1729-36.

[3] Marcucci M, Iorio A, Nobili A, Tettamanti M, Pasina L, Marengoni A, et al. Factors affecting adherence to guidelines for antithrombotic therapy in elderly patients with atrial fibrillation admitted to internal medicine wards. Eur J Intern Med 2010;21:516-23.

[4] Tulner LR, van Campen JP, Kuper IM, Gijsen GJ, Koks CH, Mac Gillavry MR, et al. Reasons for undertreatment with oral anticoagulants in frail geriatric outpatients with atrial fibrillation: a prospective, descriptive study. Drugs Aging 2010;27:39-50.

[5] Scowcroft AC, Lee S, Mant J. Thromboprophylaxis of elderly patients with AF in the UK: an analysis using the General Practice Research Database (GPRD) 2000-2009. Heart 2013;99:127-32.

[6] Lip GY, Nieuwlaat R, Pisters R, Lane DA, Crijns HJ. Refining clinical risk stratification for predicting stroke and thromboembolism in atrial fibrillation using a novel risk factor-based approach: the euro heart survey on atrial fibrillation. Chest 2010;137: 263-72.

[7] Olesen JB, Torp-Pedersen C, Hansen ML, Lip GY. The value of the CHA2DS2-VASc score for refining stroke risk stratification in patients with atrial fibrillation with a CHADS2 score 0-1: a nationwide cohort study. Thromb Haemost 2012;107:1172-9.

[8] Gage BF, Waterman AD, Shannon W, Boechler M, Rich MW, Radford MJ. Validation of clinical classification schemes for predicting stroke: results from the National Registry of Atrial Fibrillation. JAMA 2001;285:2864-70.

[9] Camm AJ, Kirchhof P, Lip GY, Schotten U, Savelieva I, Ernst S, et al. Guidelines for the management of atrial fibrillation: the task force for the management of atrial fibrillation of the European Society of Cardiology (ESC). Eur Heart J 2010;31:2369-429.

[10] You JJ, Singer DE, Howard PA, Lane DA, Eckman MH, Fang MC, et al. Antithrombotic therapy for atrial fibrillation: antithrombotic therapy and prevention of thrombosis, 9th ed.: American College of Chest Physicians Evidence-Based Clinical Practice Guidelines. Chest 2012;141:e531S-75S.
[11] Skanes AC, Healey JS, Cairns JA, Dorian P, Gillis AM, McMurtry MS, et al. Focused 2012 update of the Canadian Cardiovascular Society atrial fibrillation guidelines: recommendations for stroke prevention and rate/rhythm control. Can J Cardiol 2012;28:125-36

[12] Gage BF, Yan Y, Milligan PE, Waterman AD, Culverhouse R, Rich MW, et al. Clinical classification schemes for predicting hemorrhage: results from the National Registry of Atrial Fibrillation (NRAF). Am Heart J 2006;151:713-9.

[13] Shireman TI, Mahnken JD, Howard PA, Kresowik TF, Hou Q, Ellerbeck EF. Development of a contemporary bleeding risk model for elderly warfarin recipients. Chest 2006;130:1390-6.

[14] Pisters R, Lane DA, Nieuwlaat R, de Vos CB, Crijns HJ, Lip GY. A novel user-friendly score (HAS-BLED) to assess 1-year risk of major bleeding in patients with atrial fibrillation: the Euro Heart Survey. Chest 2010;138:1093-100.

[15] Fang MC, Go AS, Chang Y, Borowsky LH, Pomernacki NK, Udaltsova N, et al. A new risk scheme to predict warfarin-associated hemorrhage: the ATRIA (Anticoagulation and Risk Factors in Atrial Fibrillation) study. J Am Coll Cardiol 2011;58: 395-401.

[16] Nobili A, Licata G, Salerno F, Pasina L, Tettamanti M, Franchi C, et al. Polypharmacy, length of hospital stay, and in-hospital mortality among elderly patients in internal medicine wards. The REPOSI study. Eur J Clin Pharmacol 2011;67:507-19.

[17] Lip GY, Frison L, Halperin JL, Lane DA. Comparative validation of a novel risk score for predicting bleeding risk in anticoagulated patients with atrial fibrillation: the HAS-BLED (Hypertension, Abnormal Renal/Liver Function, Stroke, Bleeding History or Predisposition, Labile INR, Elderly, Drugs/Alcohol Concomitantly) score. J Am Coll Cardiol 2011;57:173-80.

[18] Olesen JB, Lip GY, Hansen PR, Lindhardsen J, Ahlehoff O, Andersson C, et al. Bleeding risk in 'real world' patients with atrial fibrillation: comparison of two established bleeding prediction schemes in a nationwide cohort. J Thromb Haemost 2011;9: $1460-7$.

[19] Breiman L, Friedman JH, Olshen RA, Stone CJ. Classification and regression trees. New York: Chapman \& Hall (Wadsworth, Inc.); 1984.

[20] Dorresteijn JA, Visseren FL, Ridker PM, Wassink AM, Paynter NP, Steyerberg EW, et al. Estimating treatment effects for individual patients based on the results of randomized trials. BMJ 2011:343.

[21] Devereaux PJ, Anderson DR, Gardner MJ, Putnam W, Flowerdew GJ, Brownell BF, et al. Differences between perspectives of physicians and patients on anticoagulation in patients with atrial fibrillation: observational study. BMJ 2001;323:1218-22.

[22] LaHaye SA, Gibbens SL, Ball DG, Day AG, Olesen JB, Skanes AC. A clinical decision aid for the selection of antithrombotic therapy for the prevention of stroke due to atrial fibrillation. Eur Heart J 2012;33:2163-71.

[23] Carlsson AC, Wandell P, Sundquist K, Johansson SE, Sundquist J. Differences and time trends in drug treatment of atrial fibrillation in men and women and doctors' adherence to warfarin therapy recommendations: a Swedish study of prescribed drugs in primary care in 2002 and 2007. Eur J Clin Pharmacol 2013;69:245-53.

[24] Sandhu R, Bakal AB, Ezekowitz JA, McAlister FA. Risk stratification schemes, anticoagulation use and outcomes: the risk-treatment paradox in patients with newly diagnosed non-valvular atrial fibrillation. Heart 2011;97:2046-50.

[25] Rash A, Downes T, Portner R, Yeo WW, Morgan N, Channer KS. A randomised controlled trial of warfarin versus aspirin for stroke prevention on octogenarians with AF (WASPO). Age Ageing 2007;36:151-6.

[26] Mant J, Hobbs R, Fletcher K, Roalfe A, Fitzmaurice D, Lip GY, et al. Warfarin versus aspirin for stroke prevention in an elderly community population with AF (the Birmingham AF Treatment of the Aged Study BAFTA): a randomised controlled trial. Lancet 2007;370:493-503.

[27] Guo Y, Lip GY, Apostolakis S. The challenge of antiplatelet therapy in patients with atrial fibrillation and heart failure. J Cardiovasc Transl Res 2013;6:388-97. 